\title{
Function algebras on the interval and circle
}

by

DAVID M. WELTS (Pittsburgh, Penn.)

Alstract. A function algebra whose maximal ideal space is the interval $I$ is local. A function algebra whose maximal ideal space is the circle $T$ is either local or antisymmetric. A strongly regular function algebra on $T$ must be $O(T)$.

Throughout this paper $A$ will denote a function algebra on a compact Hausdorff space $X$, with maximal ideal space $M_{\mathcal{A}}$ and Shilov boundary $S_{\mathcal{A}}$. If $K$ is a closed subset of $X$, the algebra of restrictions to $K$ of functions in $A$ will be denoted by $\left.A\right|_{K}$, and its uniform closure in $C(K)$ will be denoted by $A_{K}$. The unit interval and unit circle will be denoted by $I$ and $T$ respectively. We will need the definitions listed below. For additional background, see [1] or [3].

Say that a function $f$ in $O(X)$ belongs locally to $A$ if there are open sets $U_{1}, \ldots, U_{n}$ covering $X$ and functions $g_{1}, \ldots, g_{n}$ in $A$ such that the restriction of $f$ to $U_{i}$ agrees with $g_{i}$ for each $i$. $A$ is sajd to be a local algebra if it contains every function belonging locally to $A$. $A$ is called approximately normal if for any two disjoint closed subsets $K$ and $L$ of $X$ and any $r>0$, there is a function $h$ in $A$ with $|h|<r$ on $K$ and $|h-1|<r$ on $L$.

At present, there are no known examples of function algebras other than $O(I)$ or $O(T)$ with $M_{4}=I$ or $T$. In [4] it is shown that any such algebra must be approximately normal. Our main result concerns functions belonging locally to such algebras. We will make use of the following fact from [4]: if $A$ is approximately normal on $X, f \in O(X)$ and there are open sets $U_{1}, U_{2}$ covering $X$ and functions $g_{1}, g_{2}$ in $A$ with $\left.f\right|_{U_{i}}=\left.g_{i}\right|_{U_{i}}$ then $f \in A$.

THronem. If $A$ is a function algebra whose maximal ideal space is $I$, then $A$ is a local algebra.

Let $g \in O(I)$ and suppose there is an open covering of $I$ by sets $U_{1}, \ldots, U_{n}$, such that $\left.g\right|_{U_{i}}=\left.g_{i}\right|_{U_{i}}$ for some $g_{i} \in A, i=1, \ldots, n$. We may assume each $U_{i}$ is a subinterval of $I, U_{i} \cup U_{i+1} \neq \varnothing$ for $i=1, \ldots, n-1$, and $\left\{U_{1}, \ldots, U_{n}\right\}$ has no proper subcover. Since $A$ is approximately normal on $I$, we can find a function $f_{1} \in A$ agreeing with $g_{1}$ on $U_{1}$ and 
with $g_{2}$ on $U_{2} \cup \ldots \cup U_{n}$. Then $f_{1}$ agrees with $g$ on $U_{1} \cup U_{2}$. Continuing inductively, we can find functions $f_{i} \in A$ agreeing with $f_{i-1}$ on $U_{1} \cup \ldots$ $\cup U_{i}$, and with $g_{i+1}$ on $U_{i+1} \cup \ldots \cup U_{n}$, for $i=2, \ldots, n-1$. Since $f_{i}$ agrees with $g$ on $U_{1} \cup \ldots \cup U_{i+1}, f_{n-1}=g$.

Corollari. If $A$ is a function algebra whose maximal ideal space is $T$, then $A$ is either antisymmetric or local.

Suppose $g \epsilon C(T)$ is locally in $A$. Let $K$ be an arbitrary maximal set of antisymmetry for $A$. If $K=T$, then $A$ is antisymmetric. Otherwise we need to show $g \in A$, and by a result in [2] it is enough to show $\left.g\right|_{K} \in A_{K}$. Since $K \neq T$ we can find an are $J$ containing $K$. It is shown in [4.] that $J$ must be $A$-convex, so $A_{J}$ is a function algebra whose maximal ideal space is the interval $J$. Now $\left.g\right|_{J}$ is locally in $A_{J}$, and $A_{J}$ is a local algebra. Hence $\left.g\right|_{J} \in A_{J}$, and in particular, $\left.g\right|_{K} \in A_{K}$.

A function algebra $A$ on $X$ is said to be analytic if any function in $A$ vanishing on a non-empty open set in $X$ must vanish identically on $X$. It is known that an analytic function algebra on $X$ must be an integral domain, and that a function algebra on $X$ which is an integral domain must be antisymmetric on $X$. It is easy to see that an analytic function algebra is local whenever $X$ is connected. We will be able to show that if $M_{A}=T, A$ is local as long as it is an integral domain.

Lemara. Let $A$ be approximately normal on $X$. Suppose $A$ is an integral domain. If $Z$ is the zero-set of any function $f \in A$, then $X-Z^{0}$ is oonnected.

If $X-Z^{0}$ is not connected, it is the union of two disjoint non-empty compact sets $K$ and $L$. It is easy to show that $K$ and $L$ have non-empty interiors in $X$, so that $\left.f\right|_{K}$ and $\left.f\right|_{L}$ are not identically zero. Using approximate normality, we can obtain functions $g$ and $h$ in $A$ such that $\left.g\right|_{X-K}$ $=0=h_{X-L}, g$ agrees with $f$ on $K$ and $h$ agrees with $f$ on $L$. But then $g h=0$, a contradiction since $A$ is an integral domain.

THEOREM. Let $A$ be approximately normal on $T$. If $A$ is an integral domain, then $A$ is local.

Suppose $f \in O(T)$ is locally in $A$ but not in $A$. Choose a cover $\mathscr{U}$ $=\left\{U_{1}, \ldots, U_{n}\right\}$ of $T$ and functions $g_{1}, \ldots, g_{n} \in A$ such that $\left.f\right|_{U_{i}}=g_{i}$. Assume each $U_{i}$ is an arc, $U$ has no proper subcover and $\left.\left.f\right|_{V_{i} \cup V_{i} \phi A}\right|_{V_{i}} \cup V_{i}$ if $U_{i} \cap U_{j} \neq \varnothing$. Let $W_{i}=U_{i}$ together with the interiors of the zero-sets of $g_{i}-g_{i-1}$ and $g_{i}-g_{i+1}, i=1, \ldots, n$, where we understand $n+1=1$. By the above lemma, each $W_{i}$ is an arc, so if $\mathscr{V}=\left\{V_{1}, \ldots, V_{m}\right\}$ is a minimal subcover of $\left\{W_{1}, \ldots, W_{n}\right\}$, then $\mathscr{V}$ has all the properties attributed above to $\mathscr{U}$. We may assume $m=n$ and $f$ agrees with $g_{i}$ on $V_{i}$, so the interior of the zero-set of $g_{i}-g_{i+1}$ is exactly $V_{i} \cap V_{i+1}$. Let $f_{i}=\left(g_{i}-g_{i+1}\right)$ $\left(g_{i}-g_{i+1}\right)$. If $Z_{i}$ is the zero-set of $f_{i}$, then $Z_{i}^{0}$ contains $\left(V_{i} \cap V_{i-1}\right) \cup\left(V_{i} \cap V_{i+1}\right)$ but $Z_{i}^{0} \subseteq V_{i}$ by the construction of $\mathscr{V}$. Hence $Z_{i}^{0}=V_{i}$, but then $f_{1} f_{2} \ldots f_{n}$ $=0$, a contradiction.
A function algebra $A$ on $X$ is called strongly regular if any function in $A$ vanishing at a point $x \in X$ can be uniformly approximated in $A$ by functions vanishing in a neighborhood of $x$, the neighborhood depending on the function. In [5] it is shown that any strongly regular function algebra is normal, and that a strongly regular function algebra on $I$ must be $C(I)$. We can prove the corresponding result for $T$.

THEOREM. If $A$ is a strongly regular function algebra on $T$, then $A=C(T)$.

It is enough to show that for any $r>0$ and any two disjoint closed ares $K$ and $L$ of $T$, there is a function $g \in A$ with $|g|<r$ on $K,|g-1|<r$ on $L$ and $\|g\|$ bounded by some constant independent of $r$. The Shilov boundary of $A$ must be $T$, so we may assume the endpoints of $K$ are peak points for $A$, say $x$ and $y$. Choose a neighborhood $U$ of $\{x, y\}$ with $\bar{U} \cap L=\varnothing$. We can find a function $f \in A$ such that $\|f\|<2, f(x)=f(y)=0$ and $|f-1|<r$ outside $U$. Choose functions $h, k \in A$ such that $\|h-f\|<r$, $\|z-f\|<r$ and $h$ and $k$ vanish in neighborhoods $U$ and $W$ of $x$ and $y$ respectively. Now $h \%$ vanishes on $V \cup W$, is close to 1 on $L$, and $\|h k\|<5$ for sufficiently small values of $r$. Using normality, we can now obtain a function $g \in A$ vanishing on $K$ and agreeing with $h \hbar$ on $T-K$.

\section{References}

[1] A. Browder, Introduction of Funotion Algebras, 1969.

[2] I. Glicksberg, Measures orthogonal to algebras and sets of antisymmetry, Trans. Amer. Math. Soc. 105 (1962), pp. 414-435.

[3] G. Leibowitz, Lectures on Oomplex Function Algebras, 1970.

[4] D. Wilken, Approximate normality and function algebras on the interval and circle, pp. 98-111, Proc. Internat. Sympos. on Function Algebras, Tulane Univ., 1965-1966.

[5] - A Note on Strongly Regular Fiunction Algebras, Can. J. Math. 21 (1969). UNIVERSITY OF PITTSBURGH 